\title{
The Effectiveness of Isometric Contractions Compared With Isotonic Contractions in Reducing Pain For In-Season Athletes With Patellar Tendinopathy
}

\author{
Chee Vang and Alexander Niznik
}

\begin{abstract}
Clinical Scenario: Patellar tendinopathy is a common musculoskeletal disorder affecting the lower-extremities and a difficult condition to manage for athletes that are in season. To facilitate improvement in function and to decrease pain, initial treatment for patellar tendinopathy is typically conservative. Traditional interventions may include eccentric training, cryotherapy, patellar counterforce straps, oral anti-inflammatories, injectable agents, phonophoresis, iontophoresis, orthotics, therapeutic ultrasound, and extracorporeal shockwave. In addition, recent literature suggests that implementing isometric and isotonic contractions may be effective in reducing patellar tendon pain. Focused Clinical Question: How effective are isometric contractions compared with isotonic contractions in reducing pain for in-season athletes with patellar tendinopathy? Summary of Key Findings: Implementation of isometric and isotonic exercises statistically reduced pain levels in the short term of 4 weeks for in-season athletes; however, isometric contractions provided statistically greater pain relief immediately for up to 45 minutes postintervention compared with isotonic contractions. Clinical Bottom Line: Current evidence supports the use of isometric and isotonic contractions to reduce pain for in-season athletes with patellar tendinopathy. Based on the reviewed literature, clinicians should consider utilizing heavy loaded isometrics or progressive heavy loaded isotonic exercises, which showed reduction in pain levels immediately after intervention and at 4-week follow-up for both intervention groups. Isometric contractions appear to provide greater pain relief immediately after intervention. Strength of Recommendation: There is Grade B evidence from 2 level 2 randomized controlled trials and 1 level 3 randomized crossover study supporting the use of isometric and isotonic contractions to reduce patellar tendon pain for in-season athletes.
\end{abstract}

Keywords: physical therapy, isotonic, exercise, load, tendon

\section{Clinical Scenario}

Patellar tendinopathy is a common musculoskeletal disorder affecting the lower-extremities and a difficult condition to manage for athletes that are in season. ${ }^{1-3}$ Pain is a common factor that may negatively affect an athlete's ability to train with optimal volume and intensity and maximize performance for their sport. ${ }^{1-3}$ To facilitate improvement in function and to decrease pain, initial treatment for patellar tendinopathy is typically conservative. Traditional interventions may include eccentric training, cryotherapy, patellar counterforce straps, oral anti-inflammatories, injectable agents, phonophoresis, iontophoresis, orthotics, therapeutic ultrasound, and extracorporeal shockwave. ${ }^{2-6}$ Exercise and tendon loading appear to demonstrate positive effects in histological changes and reduction in pain perception. Eccentric exercises have been widely utilized to treat patellar tendinopathy and are effective in maximizing an athlete's function over time but are typically poorly tolerated due to an increase in pain levels in the first 2 to 4 weeks and have shown either no benefit or worse outcomes for in-season athletes along with poor adherence to exercise regimen due to pain increase. ${ }^{2,3}$ This pain-induced loading technique may lead to cortical reorganization, which, in turn, may contribute to suboptimal motor control, contributing to ongoing tendon pain. In addition, recent literature suggests implementing

Vang is with Twin Cities Orthopedics, Eagan, MN, USA. Niznik is with Twin Cities Orthopedics, Burnsville, MN, USA. Vang (cheevang24@gmail.com) is corresponding author. isometric and isotonic contractions as these may be effective alternatives in reducing patellar tendon pain for in-season athletes due to better pain tolerance, which may help to optimize their performance for sport. ${ }^{1-3}$ Isometric exercise has been shown to affect cortical changes with antinociceptive effects including a release of intracortical inhibition, which was associated with pain reduction. ${ }^{1}$ Pain during exercise has been a primary contributor to suboptimal performance and limiting training and sport participation for in-season athletes. Therefore, this critically appraised topic was conducted to determine the extent to which current evidence supports the use of isometric contractions compared with isotonic contractions to reduce pain for in-season athletes with patellar tendinopathy.

\section{Focused Clinical Question}

How effective are isometric contractions compared with isotonic contractions in reducing pain for in-season athletes with patellar tendinopathy?

\section{Summary of Search, "Best Evidence" Appraised, and Key Findings}

- The literature was searched for studies of level 3 evidence or higher that directly compared isometric contractions with isotonic contractions for patellar tendinopathy.

- The literature search returned 4 possible studies related to the clinical question; 3 studies met the inclusion criteria. 
- Two level 2 studies and 1 level 3 study compared the effects of pain levels with isometric contractions versus isotonic contractions among individuals with patellar tendinopathy.

- Three studies reported a statistically significant reduction in pain with isometric and isotonic contractions in the short term of 4 weeks ${ }^{1-3}$; however, isometric contractions provided statistically greater pain relief immediately, which remained up to 45 minutes postintervention compared with isotonic contractions. $^{1}$

\section{Clinical Bottom Line}

Current evidence supports the use of isometric and isotonic contractions to reduce pain for in-season athletes with patellar tendinopathy. ${ }^{1-3}$ Based on the reviewed literature, clinicians should consider utilizing heavy loaded isometrics or progressive heavy loaded isotonic exercises, which showed reduction in pain levels immediately after intervention and at 4-week follow-up for both intervention groups. ${ }^{1-3}$ Isometric contractions appear to provide greater pain relief immediately for up to 45 minutes after intervention compared with isotonic contractions when utilizing a single-leg decline squat as a pretest and posttest to measure pain response. ${ }^{1}$

\section{Strength of Recommendation}

There is Grade B evidence from 2 level 2 randomized controlled trials $^{2,3}$ and 1 level 3 randomized crossover study ${ }^{1}$ supporting the use of isometric and isotonic contractions to reduce patellar tendon pain for in-season athletes.

\section{Search Strategy}

\section{Term Used to Guide Search Strategy}

- Patient/client group: patellar tendinopathy

- Intervention/assessment: isometric exercise

- Comparison: isotonic exercise

- Outcome: pain

\section{Sources of Evidence Searched}

- PubMed

- Google Scholar

- SPORTDiscus

- EBSCOhost

- CINAHL

- MEDLINE

- Additional resources obtained via review of reference list and manual search

\section{Inclusion and Exclusion Criteria}

\section{Inclusion}

- Level 3 evidence or higher

- Subjects were clinically diagnosed with patellar tendinopathy

- Studies that directly compared isometric contractions to isotonic contractions
- Subjects were allowed to continue their sport during the intervention period

- Limited to English language

- Limited to humans

- Limited to the past 10 years (2009-2019)

\section{Exclusion}

- Subjects with other pathological conditions of the lowerextremity

- Subjects with previous surgery to the lower-extremity

- Subjects who received a corticosteroid injection within the past 12 months

\section{Results of Search}

Four studies were retrieved; however, only 3 studies that met the criteria requirements were selected for review. ${ }^{1-3}$ These are summarized in Table 1. All 3 studies compared isometric contractions with isotonic contractions for pain relief for in-season athletes with patellar tendinopathy.

\section{Best Evidence}

Three studies were identified as the best evidence for this critically appraised topic (Table 2). Two articles were level 2 randomized controlled trials, and 1 article was a level 3 randomized crossover study based on the Oxford Levels of Evidence 2011.

\section{Implications for Practice, Education, and Future Research}

In each of the 3 studies reviewed, in-season athletes with patellar tendinopathy demonstrated statistically significant reductions in pain levels in response to isometric and isotonic contractions immediately after intervention and at 4-week postintervention. ${ }^{1-}$ 3 One of the 3 studies' results displayed significant pain reduction that was greater for subjects in the isometric group, ${ }^{3}$ whereas another study indicated that pain levels remained decreased 45 minutes only after the isometric contractions. ${ }^{1}$ Therefore, both isometric and isotonic contractions are an effective intervention to reduce patellar tendon pain to allow athletes to continue play while in-season without modification of current training loads. However, neither study compared these contractions types with other alternative interventions, so the authors cannot determine if they are equally if not more effective.

The etiology of load-induced patellar tendinopathy is multifactorial and involves tendon overload through tension, compression, or friction, which may lead to a series of tendon injury, repair, and inflammation, ${ }^{7}$ which may cause pain and dysfunction. ${ }^{1-7}$ Nonoperative management has typically included the use of

\section{Table 1 Summary of Study Designs of Retrieved Articles}

\begin{tabular}{lll}
\hline Levels of evidence & \multicolumn{1}{c}{ Study design } & \multicolumn{1}{c}{ Author } \\
\hline 2 & Randomized controlled trial & Van ark et $\mathrm{al}^{3}$ \\
& & Rio et $\mathrm{al}^{2}$ \\
3 & Randomized crossover & Rio et $\mathrm{al}^{1}$ \\
\hline
\end{tabular}




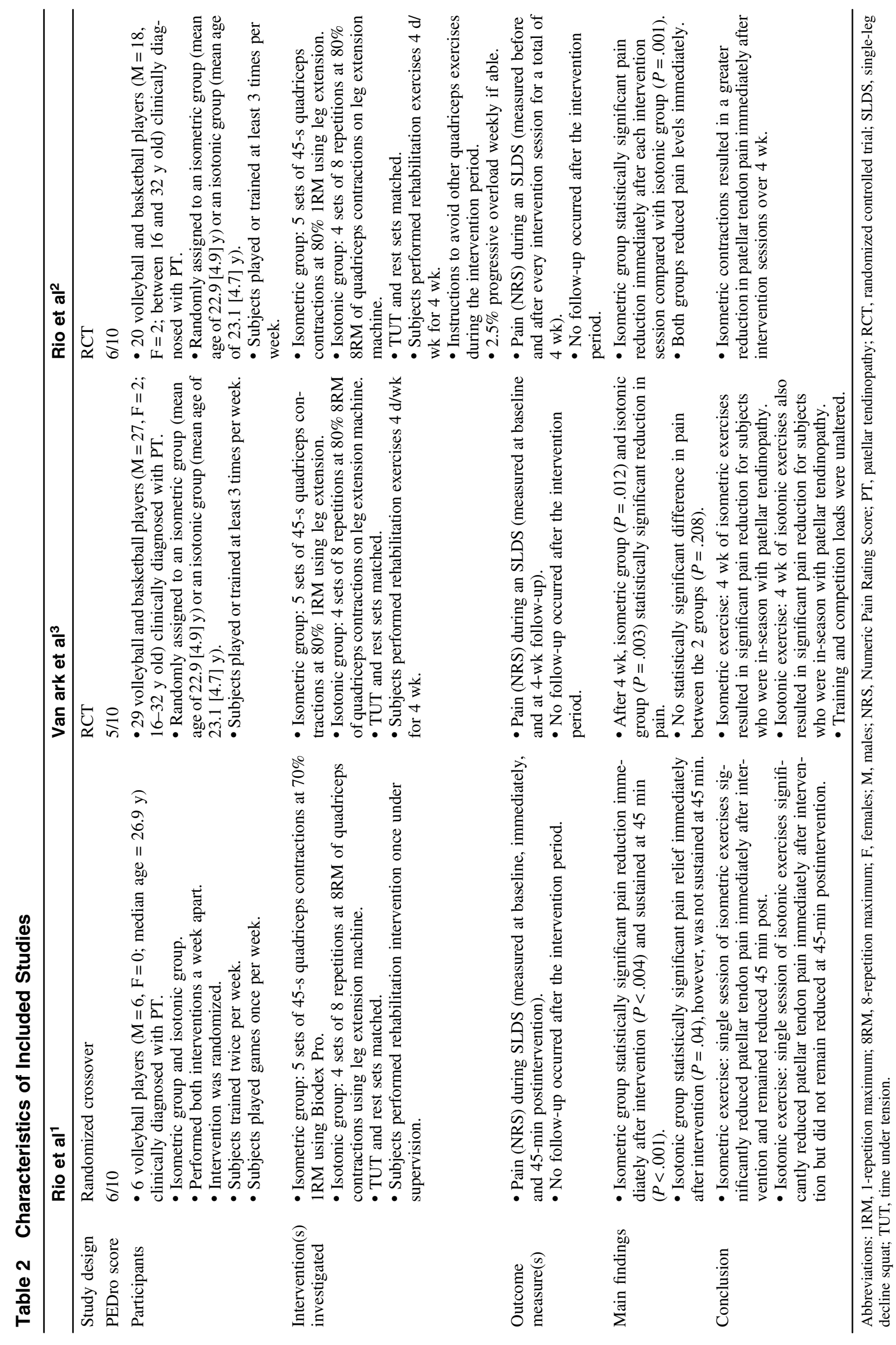


eccentric contractions performed on a decline board, ${ }^{2-5}$ injection therapy, and extracorporeal shockwave. ${ }^{3}$ Patellar tendinopathy is a common pathology occurring in individuals who perform explosive movements that load the quadriceps and is prevalent in jumping sports including volleyball and basketball. ${ }^{2}$

Exercise and tendon loading are suggested as the best intervention to decrease patellar tendon pain and maximize function; however, the mechanisms are not well understood. Current literature suggests that loading the tendon through exercise affects the tendon matrix ${ }^{3}$ and reduces pain perception. ${ }^{1-3}$ In addition, patellar tendinopathy may be associated with nervous system sensitization, ${ }^{8,9}$ which may be addressed through various exercise prescriptions. It is suggested that isometric and isotonic exercises may be better tolerated to still allow tendon adaptation through loading while minimizing pain increase, which may facilitate an athlete's ability to train and maximize their sport performance while in season. For isometric contractions, 5 sets of 45 -second repetitions at $70 \%$ to $80 \%$ maximum voluntary contractions should be performed on a leg extension machine with the knee flexed to $60^{\circ} .{ }^{1-3}$ Isotonic contractions should be completed with 4 sets of 8 repetitions at the athlete's 8-repetition maximum on a leg extension machine. ${ }^{1-3}$

Future studies may investigate the therapeutic mechanisms of isometric and isotonic contractions in individuals affected by patellar tendinopathy. Intervention period was 4 weeks or less, therefore, there may be benefit from longer term studies beyond 4 weeks comparing isometric and isotonic contractions. Next, adherence to specific loading programs was not tracked as programs were meant to compliment the athletes' schedules. The authors in the reviewed studies assumed that the athletes consistently adhered to the strengthening programs due to finding improvements in pain levels and function at the end of the testing period. More supervised sessions may allow for program adjustments and further facilitate adherence. Finally, sample sizes were small, which may affect the external validity of implementation of isometric and isotonic contractions, and although these exercises seem to have a role in rehabilitating patellar tendinopathy, the extent of their effects require further study. Due to the apparent benefits of isometric and isotonic contractions for in-season athletes with patellar tendinopathy, future research should consider evaluation of the impact of isometric and isotonic contractions on other knee pathologies.

\section{References}

1. Rio E, Kidgell D, Purdam C, et al. Isometric exercises induces analgesia and reduces inhibition in patellar tendinopathy. Br J Sports Med. 2015;49(19):1277-1283. PubMed ID: 25979840 doi:10.1136/ bjsports-2014-094386

2. Rio E, Purdam C, Girdwood M, Cook JL. Isometric exercise to reduce pain in patellar tendinopathy in-season; is it effective on the road? Clin J Sport Med. 2017;29(3):188-192. PubMed ID: 31033611 doi:10.1097/JSM.0000000000000549

3. Van Ark M, Cook JL, Docking SI, et al. Do isometric and isotonic exercise programs reduce pain in athletes with patellar tendinopathy inseason? A randomised clinical trial. J Sci Med Sport. 2015;19(9):702706. PubMed ID: 26707957 doi:10.1016/j.jsams.2015.11.006

4. Purdam CR, Johnsson P, Alfredson H, Lorentzon R, Cook JL, Khan KM. A pilot study of the eccentric decline squat in the management of painful chronic patellar tendinopathy. Br J Sports Med. 2004;38(4):395-397. PubMed ID: 15273169 doi:10.1136/bjsm.2003. 000053

5. Zwerver J, Bredeweg SW, Hof AL. Biomechanical analysis of the single-leg decline squat. Br J Sports Med. 2007;41(4):264-268. PubMed ID: 17224441 doi:10.1136/bjsm.2006.032482

6. Reinking MF. Current concepts in the treatment of patellar tendinopathy. Int J Sports Phys Ther. 2016;11(6):854-866. PubMed ID: 27904789

7. Scott A, Backman LJ, Speed C. Tendinopathy: update on pathophysiology. J Orthop Sports Phys. 2015;45(11):833-841. PubMed ID: 26390273 doi:10.2519/jospt.2015.5884

8. Van Wilgen CP, Konopka KH, Keizer D, Zwerver J, Dekker R. Do patients with chronic patellar tendinopathy have an altered somatosensory profile? A quantitative sensory testing (QST) study. Scand J Med Sci Sports. 2013;23(2):149-155. PubMed ID: 22092832 doi:10. 1111/j.1600-0838.2011.01375.x

9. Jayaseelan DJ, Weber MJ, Jonely J. Potential nervous system sensitization in patients with persistent lower extremity tendinopathies: 3 case reports. J Orthop Sports Phys. 2019;49(4):272-279. PubMed ID: 30759356 doi:10.2519/jospt.2019.8600 